\title{
EvacSim: A Simulation Model of Occupants with Behavioural Attributes in Emergency Evacuation of High-Rise Building Fires
}

\author{
LEONG S. POON ${ }^{1}$ \\ The Centre for Environmental Safety and Risk Engineering (CESARE) \\ Victoria University of Technology (VUT) \\ P.O. Box 14428, Melbourne Mail Centre, Melbourne, Victoria 3000, Australia
}

\begin{abstract}
EvacSim is a building evacuation model which is developed based on a discrete event simulation concept. It can simulate a complex variety of human behavioural activities, both deterministically and probabilistically or a combination of both. An occupant may be modelled to respond to cues from the physical environment, or to interact with other occupants, in a manner which depends upon the level of severity perceived by the occupant. A wide variety of situations can be modelled including closed or locked doors, queuing at exits, controlled evacuation sequence, backtracking due to blocked exits, tracking of occupant movements and development of bottlenecks. Stairs, elevators and disabled occupants may also be modelled. A unique feature is the modelling of wardens to perform room-to-room searches and to control the evacuation of floor levels. EvacSim forms part of a greater model for assessing life safety in high-rise building fires.
\end{abstract}

KEYWORDS: Evacuation model, simulation, human behaviour

\section{INTRODUCTION}

There are a number of models developed for simulating emergency evacuation. EXITT [1] has a number of deterministic decision rules programmed in the model for occupants in residential fires but its applicability is limited to small family dwellings. BFIRES [2] simulates the movement of people within building enclosures in response to physical cues in the environment at regular time intervals. Human behaviour is modelled

1 The author is presently seconded to CESARE, VUT from BHP Research - Melbourne Laboratories (245-273, Wellington Road, Mulgrave, Victoria 3170, Australia) for a threeyear period commencing from August 1992 under a joint BHP/VUT sponsorship scheme. 
through three processes: perception, interpretation and response, but congestion and queuing are not modelled. EMBER [3] simulates the movement of both occupants and smoke. The modelling of human behaviour, however, is limited to predetermined egress and rescue policies. BGRAF [4] simulates the decision making processes during emergency egress and incorporates greater emphasis in the building details and way-finding characteristics of occupants. Its processing time however is considerable and is not suitable for repetitive runs normally required for fire safety assessments. EXIT89 [5] has been developed to overcome the limitations of EXITT but it does not explicitly consider human behaviour of occupants. Although the models mentioned above vary in their approaches and scope, none of them considers a managed evacuation sequence which is practised in high-rise buildings. This paper introduces a model which has been developed to address the abovementioned limitations.

EvacSim is a discrete event simulation model for analysing the emergency evacuation of high-rise buildings in fire. It has the capability to model a large population but still considers human behavioural activities on an individual level. In addition, it incorporates a flexible approach to modelling human behaviour by allowing the modeller to specify the behavioural activities which are appropriate to the type of building occupancy considered. A complex variety of human behavioural activities can be simulated, both deterministically and probabilistically or a combination of both. An occupant may be modelled to respond to cues from the physical environment, or to interact with other occupants, in a manner which depends upon the level of severity perceived by the occupant.

EvacSim forms part of a larger life safety model for assessing the safety of occupants in buildings due to the effects of fire. The life safety model is intended to incorporate other specific models such as fire growth and spread, effects of exposure of occupants to toxic gases, building subsystems (sprinklers, smoke management, detectors, etc.) and fire brigade intervention. EvacSim has been designed to accommodate the future integration with these models as they become developed or incorporated into the life safety model.

\section{MODEL DESCRIPTION}

Unlike many other evacuation models, EvacSim is not based on a network of arcs and paths. Instead it uses details similar to an architectural floor-plan of a building to define the connectivity between enclosures and between floor levels. Wall, floor and ceiling boundaries are defined independently with openings specified, if any, in the boundary. Material layers for the boundaries (including doors and windows) and their corresponding physical and chemical properties may also be specified although they will only be used when EvacSim is integrated with a fire model. Enclosures are defined by nominating the boundaries which enclose it, hence enabling enclosures with more than six planar boundary surfaces to be defined.

\section{Evacuation Management}

EvacSim enables a wide range of evacuation management strategies to be modelled and hence assessed. The evacuation of an entire building or a selective part of it may be modelled using either a warden system or an emergency warning system. 
The use of wardens for modelling evacuation in buildings is an innovative feature in EvacSim. Although wardens are an integral part of many evacuation management strategies incorporated in high-rise buildings, they are not presently incorporated in other evacuation models. The actions of wardens in response to the fire, alike actions of other occupants in the building, are determined by the user. However, the unique feature is the ability of the warden to perform a room-to-room search for occupants on the floor level. On the level of fire origin, the warden will instruct the occupants to immediately vacate the floor. On other levels, the warden will hold the occupants at exit points until instructions are received from a master warden to commence evacuation into the stairs. The warden will communicate to the master warden when the floor is cleared of occupants evacuating by stairs and is always the last occupant to leave the floor.

Alternatively, instead of wardens, floors may also be selectively evacuated using an emergency warning and intercommunication system (EWIS). The decision to evacuate however, will always depend upon how the responses have been defined for the occupant to the perceived cues, which in this case will include the information broadcasted.

\section{Human Behaviour}

It is essential to model the activities of occupants during building evacuation in order to obtain realistic results for evacuation times. The obvious difficulty in modelling human behaviour is the unpredictability associated with the decision-making of occupants when responding to a set of stimuli.

The approach adopted in EvacSim avoids the need to incorporate a specific human behavioural model. Instead, the resulting action of an occupant in response to a particular cue is obtained from a specified assembly of scales and matrices as shown in Figure 1. The data may be structured to suit a particular behavioural model or obtained from results of experimental investigations to reflect the occupant characteristics of the building considered. The advantage of this approach is that EvacSim only needs to consider the time and spatial variations of each individual occupant due to an activity that is determined as a dynamic function of a range of cues that are modelled. The complex behavioural interactions which are structured into the defined activities of the occupant are therefore indirectly avoided by EvacSim. The input data for modelling human behaviour are categorised as follows:

(a) Severity Scale. The severity scale represents an arbitrary number of levels of severity to be modelled. Typical levels of severity are low, medium and high. Each level of severity corresponds to a particular range of responses described in (c) below.

(b) Physical Scale. The physical scale represents the range of a physical cue perceived by an occupant and encompasses the range of the severity scale defined previously. Typical physical scales are smoke obscuration level and air temperature. Each scale is divided into subranges such that each subrange corresponds to a particular level of severity on the severity scale. For example, air temperatures between $30-40^{\circ} \mathrm{C}$ may be designated as low severity on the severity scale, $40-50^{\circ} \mathrm{C}$ as medium severity and $50-100^{\circ} \mathrm{C}$ as high severity. Air temperatures below $30^{\circ} \mathrm{C}$ are ignored, whilst air temperatures above $100^{\circ} \mathrm{C}$ correspond to high severity. 


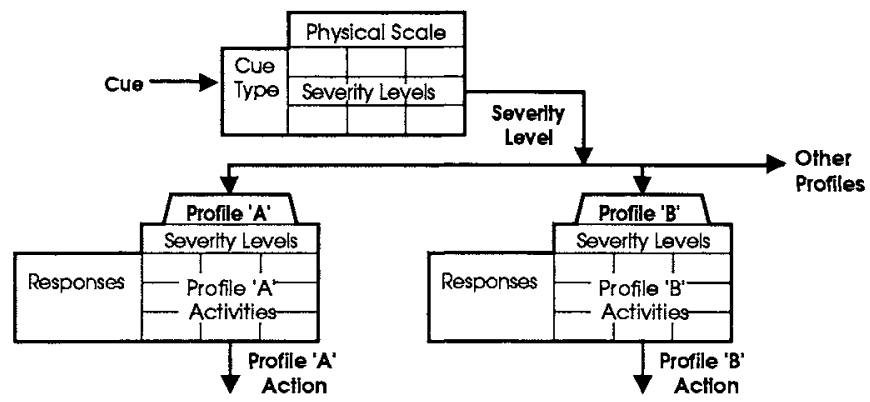

\section{FIGURE 1. Modelling Individual Occupant Actions in Response to Incoming Cues}

(c) Occupant Responses. The occupant responses are a probabilistic function of the perceived level of severity. Each response is probabilistically associated with a series of activities. The relationship between the responses, activities and the perceived levels of severity is defined in the Response Profile Table (see (g) below). Typical responses include Seek, Warn, Protect and Ignore. Typical activities for a Seek response include Investigate fire source, Get extinguisher, Return to fire source, Search for others and Seek exit.

(d) Physical Attributes. The physical attributes of an occupant are currently defined by the following parameters:

$$
\begin{aligned}
& V_{\operatorname{maxH}}-\text { The maximum horizontal travelling speed }(\mathrm{m} / \mathrm{s}) . \\
& V_{\max s}-\text { The maximum travelling speed along the stairs }(\mathrm{m} / \mathrm{s}) . \\
& A_{o} \quad \text { - The plan area occupied by an occupant }\left(\mathrm{m}^{2}\right)
\end{aligned}
$$

$V_{\max H}$ and $V_{\max }$ are used to determine the variation of travelling speed as a function of density (Figure 2). Typical values for an able-bodied occupant are as follows: $V_{\max H}=1.4, V_{\max s}=$ 0.9 (varies with the stair step profile) and $A_{o}=0.3$. These may be varied to suit slower individuals or disabled persons with wheelchairs.

(e) Building Knowledge. Presently, two categories of familiarity in a building are defined. These are exit familiarity and floor layout familiarity. Exits that are normally used in travel routes out of a building are designated as familiar exits. An occupant will only select a familiar exit unless none is available or all are blocked by fire. If an occupant is familiar with the floor layout then all possible exits are assumed to be familiar exits.

Building knowledge is assumed to be temporarily shared amongst all occupants in an enclosure as a simple means of modelling communication between occupants in the enclosure. Hence an unfamiliar occupant will select an exit assuming the knowledge of the most familiar occupant in the enclosure at the time. If the last familiar occupant was to leave the enclosure, then the remaining occupants who have already queued up at the particular exit will continue to move through the exit point. All new occupants coming into the enclosure, however, will select exits to their best available knowledge shared amongst the occupants present in the enclosure at the time. 
(f) Occupant Profile. The occupant profile is simply a record containing a particular physical attribute and a particular building knowledge attribute. An occupant profile is defined for each distinguishable category of occupants in a building. Examples of typical occupant profiles are Wardens, Residents, Visitors and Disabled.

(g) Response Profile Table. The Response Profile Table (RPT) is a matrix which associates the responses and their corresponding activities in relation to the severity scale for a particular occupant profile. An entry to the RPT for a warden may appear as shown in Table 1. In addition, each response and activity has the following attributes:

$$
\begin{array}{ll}
\text { Response: } & \text { weighting, repeatability. } \\
\text { Activity: } & \text { weighting, preparation time, response time, repeatability. }
\end{array}
$$

When more than one response or activity is specified, the weightings are used to determine the choice on a probabilistic basis. Otherwise, the weighting is ignored and the resulting response or activity becomes a deterministic reaction to the cue. A response or an activity may be repeated by setting the repeatability switch.

An activity without a weighting will follow the preceding activity upon its completion. A continuous sequence of activities can therefore be modelled, initiated by the first activity in the sequence. Each activity has a preparation time prior to executing the activity and a response time (which excludes travel time) at the end of the activity.

Duplicate activities may be defined for different responses. An occupant remembers the activities that have been carried out irrespective of which response the activity came from. Up to 8 activities per response and 8 responses to a perceived level of severity are currently imposed. A change in response can be triggered by a change in the perceived severity level at any point in time.

TABLE 1. Sample Response Profile Table Entry for a Warden

\begin{tabular}{|l|l|l|l|}
\hline Profile & Severity Level & Response & Activities \\
\hline Warden & Low & Seek & Fire $\rightarrow$ Extinguisher $\rightarrow$ Fire $\rightarrow$ Others $\rightarrow$ Exit \\
\cline { 3 - 4 } & & Warn & Activate Alarm $\rightarrow$ Others $\rightarrow$ Fire Brigade \\
\cline { 2 - 4 } & Medium & Seek & Fire $\rightarrow$ Others $\rightarrow$ Exit \\
\cline { 3 - 4 } & Warn & Activate Alarm $\rightarrow$ Others $\rightarrow$ Fire Brigade \\
\cline { 3 - 4 } & Protect & Close Door \\
\cline { 2 - 4 } & High & Seek & Exit \\
\hline
\end{tabular}

(h) Communication. In addition to the simple means of modelling the sharing of building knowledge between occupants within an enclosure, the sharing of fire knowledge is also modelled simultaneously. In this case, fire knowledge is simply modelled as the level of severity which initiated the occupant responses. Hence, an occupant with a higher level of fire knowledge (or level of severity) will transfer this knowledge to other occupants in the enclosure with lower levels. A communication time is allowed for processing this event. 
An exception to this means of communication occurs when a floor warden is conducting a room-to-room search. In this situation, occupants are forced to adopt a 'Seek Exit' activity, after communicating with the floor warden.

\section{Occupant Movement}

Initiation of Movement. All occupants are assumed to be initially inactive until an appropriate cue is received to initiate an activity. An activity may or may not cause movement. An occupant may also be modelled to perceive a particular cue to be ambiguous and ignores it, although another occupant receiving the same cue may respond differently. Responses to cues are defined in the Response Profile Table described earlier.

Travel Speed. The travel speed of an occupant is normally modelled as the variation of walking speed with occupant density. Many models express a maximum unrestricted walking speed at low density values which decreases as a function of increasing density. The maximum unrestricted walking speed predicted by most models vary from $1.2 \mathrm{~m} / \mathrm{s}$ to $1.4 \mathrm{~m} / \mathrm{s}$, although Predtechenskii and Milinskii [6] suggest a maximum speed of $0.95 \mathrm{~m} / \mathrm{s}$. The rate of reduction in walking speed with density varies from a linear relationship (Pauls [7], Nelson and MacLennan [8]) to a fourth degree polynomial (Predtechenskii and Milinskii). Considering that the occupant density itself varies continuously over time and space, and that the walking pace may be influenced by other factors such as the level of anxiety, obtaining an accurate prediction of walking speed is therefore questionable.

Rather than adopting a specific walking speed model, EvacSim uses a variable bilinear travel speed model. This is similar to an invariable bilinear model proposed by Nelson and MacLennan based on works by Fruin [9], Pauls and Predtechenskii and Milinskii. The variable bilinear travel speed model is defined by the following parameters (Figure 2):

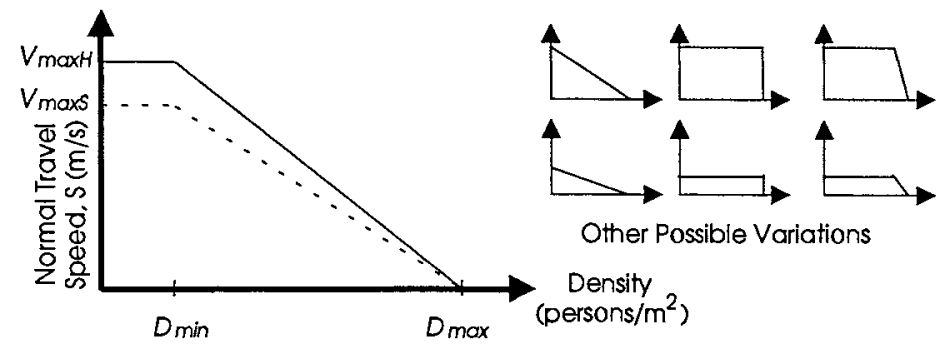

FIGURE 2. Definition of Variable Bilinear Travel Speed Model

$S \quad$ - The travel speed under normal conditions.

$D_{\min }$ - The density below which the occupant travels at the maximum speed.

$D_{\max }$ - The density at which the occupant is immobilised.

The calculation of density is made at each instant of time that an occupant commences movement to another location, and is the ratio of moving occupants to the egressable area (i.e. the area not occupied by occupants already in queues and other objects in the enclosure). 
Movement for Disabled Occupants. Travel speeds for occupants with disabilities, e.g. wheelchairs and crutches, are also based on the variable bilinear travel speed model using appropriate values of $V_{\max }$ and $V_{\operatorname{maxs}}$. Disabled occupants may be forced to use elevators by setting $V_{\max }=0$. Obstructions to overtaking of disabled occupants in wheelchairs or crutches along narrow corridors are not presently modelled.

Travel Routes. The movement of occupants in an enclosure adopts the 'L-shaped approach to exit' introduced by Takahashi et al [10] where movement is assumed to occur in an orthogonal path towards an exit due to obstruction by furniture or other objects in the enclosure. EvacSim presently assumes walls to be orthogonally aligned to the floor plan coordinates and hence the travel distance $d$ from point $\left(x_{A}, y_{A}\right)$ to point $\left(x_{B}, y_{B}\right)$ is calculated as follows:

$$
d=\left|x_{A}-x_{B}\right|+\left|y_{A}-y_{B}\right|
$$

Exit Choice. If an enclosure has a number of exits, the choice of an exit by an occupant in the enclosure depends upon the following factors:

(a) the orthogonal distance between the occupant and the exit;

(b) the length of the queue at the exit, if any;

(c) whether the exit is locked or not;

(d) the familiarity of the occupant with the exit;

(e) the familiarity of the occupant with the floor layout;

(f) whether the exit is a designated exit, i.e. with proper EXIT signage;

(g) whether the exit is blocked by the effects of fire; and

(h) the mode of the occupant, i.e. whether the occupant is evacuating the building or going to another part of the building (e.g. seeking fire source, another occupant, fire extinguisher, etc.).

Almost all of the above factors are essentially local to the conditions within the enclosure itself. The exception is factor (h) for a non-evacuating occupant where the conditions are more global in nature. In this case, additional distances are calculated from the exit points to the destination point to determine the minimum total travel distance.

In a situation whereby the occupant is trapped, i.e. all exits leading out of the enclosure are blocked, the occupant will then be set to seek refuge in locations away from the impending danger. Backtracking is therefore modelled under such conditions. Factors with relate to the effects of fire such as visibility of exits are not yet incorporated.

Width Restrictions. The flow of occupants is restricted at points where the widths are narrowed. For simplicity, width restrictions are only checked at exit points such as doors. When an occupant arrives at an exit point which is opened and is not being used then no restriction to travel is assumed, i.e. the occupant passes through the exit point without delay. If a queue is formed at an exit point of effective width, $W_{e}$, then the flow rate $F$ for calculating the time to discharge the queue is calculated using the flow equation from Nelson and MacLennan [8]:

$$
F=S D W_{e}
$$

The density $D$ at the exit is chosen such that the flow rate $F$ is maximised. 
Queuing. Queues are formed on a first-in-first-out basis, with the exception of the floor warden who always remain at the end of a queue until all occupants are evacuated. The occupants are therefore discharged according to their order in the queue. If the adjoining enclosure is full and the occupant attempting to enter it cannot proceed, the event will be postponed by $t$ Wait seconds before another attempt to enter the adjoining enclosure is made. The following causes for the formation of queues are modelled:

- The exit is blocked due to a temporarily closed or locked door;

- The adjoining enclosure is filled to capacity;

- The rate of occupant arrival to the queue exceeds the rate of discharge from the queue.

Elevators. Occupants with disability may be evacuated using elevators designed for fire evacuation if descent by stairs is not a feasible option. Priority to enter an elevator is based on a queuing rule as for the stairs (i.e. first-in-first-out basis). However, occupants leave the elevator in the reverse order (i.e. last-in-first-out basis). The following modes related to the use of elevators for evacuation are presently modelled:

- Call A request is made to use the elevator.

- Ascend The elevator travels towards the level where a request was initiated.

- Load The elevator is loading occupants.

- Wait The duration between arrival of occupants.

- Descend The elevator travels towards the discharge level.

- Unload The elevator is unloading occupants.

- Free The elevator is idle.

\section{MODEL VALIDATION AND ILLUSTRATION}

The validation of the model is planned in three stages. The first stage involves validation with hydraulic (sometimes referred to as physical-sciences or ball-bearing type) models. This process ensures that the movement and queuing theory is accurate, but not necessarily correct. The second stage of the validation checks the correctness of the model with actual data compiled from experiments. This stage will also provide enumerated data on human behavioural activities to be used in the simulation. The third stage involves validation with actual fire incidents using the human behavioural data gathered in stage 2 of the validation process. The last stage is also an attempt to validate the accuracy of the enumerated data on human behavioural activities used in Stage 2. At the time of writing this paper, only stage 1 of the validation is complete. Details of the validation will be published at a later date when all stages of the validation process are completed. A simple example will be presented instead to illustrate the human behavioural features described earlier.

Consider a two storey building with floor layouts as shown in Figure 3. For brevity, only four occupants distributed as shown on Level 2 are modelled and Level 1 is assumed to be vacant. Four profiles are defined, each with one level of severity, SevLvA, and one corresponding response, Seek. The data entries for the Response Profile Table for each profile are as follows: 
:SevLvA, $R=$ Seek: $1: 1, A=F$ ire $: 3: 5: 5: 0 ;$ Exit $: 0: 0: 0: 1$

Warden

:SevLvA, $R=$ Seek: $1: 1, A=F$ ire $: 3: 5: 5: 0 ;$ ExtEquipment $: 0: 0: 5: 0 ; F i$ re: $0: 0: 5: 0$;

others : $0: 0: 5: 0 ;$ Exit :0:0:0:1

Resident

: SevLvA, R=Seek: $1: 1, A=\operatorname{Exit}: 1: 5: 0: 1$

Visitor

:SevLvA, $R=$ Seek: $1: 1, A=\operatorname{Exit}: 1: 0: 0: 1$

All of the actions following the Seek response are modelled to occur in sequence by assigning a zero weighting to the actions following the initiating action (which has a non-zero weighting). Occupants with Resident and Visitor profiles will decide to evacuate immediately upon becoming aware of the fire. Occupants having a Management profile will search for the fire before deciding to evacuate. Wardens will decide to search for the fire, get a fire extinguisher from a specified position on the floor, return to the fire source to fight the fire, search for other occupants and order them to evacuate immediately, inform the master warden that the floor is cleared of occupants evacuating by stairs and then exit the building. Occupant profiles are assigned to the numbered occupants as follows: 1=Management, $2=$ Visitor, $3=$ Resident and $4=$ Warden.

The initiating cue is assumed to be a rise in air temperature due to a fire occurring in Enclosure 2 of Level 2. The building is not alarmed and hence the only cue is heat due to the air temperature in Enclosure 2. With only one level of severity, the Resident occupant recognises the cue when the air temperature exceeds the lower limit on the physical scale and decides to evacuate after 5 seconds preparation time. The communication time for the Warden and occupants is assumed to be 5 seconds. All doors are assumed to be open except for opening 7 which leads to the outside of the building from the stairwell at ground level. Opening 7 is assumed to be locked with a simple latch and that an occupant will take 2 seconds to unlock and open it.
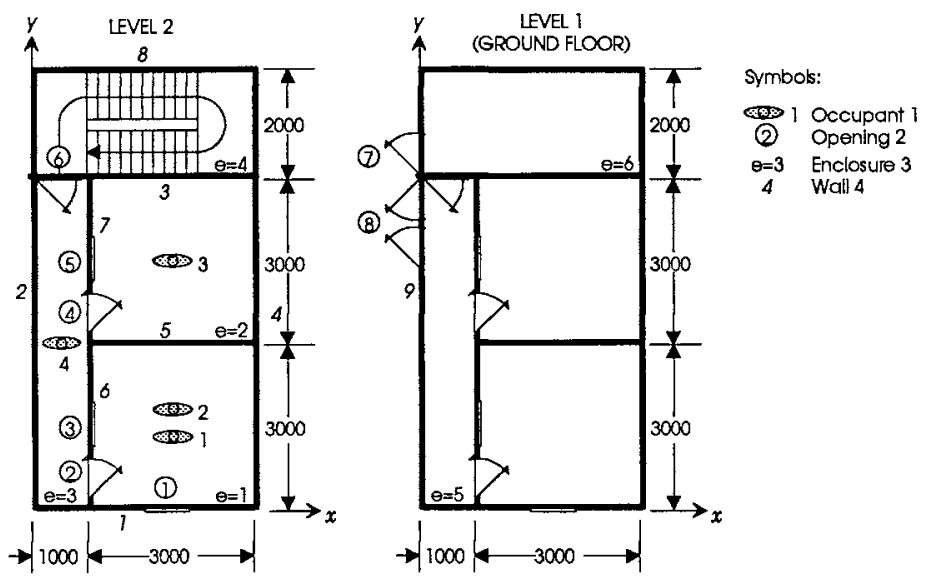

FIGURE 3. Illustrative Example 
The sequence of events which unfold in the simulation is as follows:

$0.0 \mathrm{~s}:$ Simulation starts . . 11:01:39.81

(Occupant 3 discovers fire in enclosure)

5.0s: [12 e $2 \circ 3 \times 2500$ y $4500 \mathrm{p}$ 4] going to exit opening 4

$7.1 s$ : $[12$ e $2 \circ 3 \times 1000$ y3500 p 4] reached exit opening 4

7.1s: $[12$ e $3 \circ 3 \times 1000$ y3500 p 4] tells occupant 4 (Warden) of fire

12.1s: [12 e $3 \circ 3 \times 1000$ y3500 p 4] going to exit opening 6

14.6s: [12 e $303 \times 500$ y6000 p 4] reached exit opening 6

14.6s: [12 e 4 o $3 \times 500$ y6000 p 3 ] going down the stairs

17.1s: [12 e $304 \times 500$ y $3000 \mathrm{p}$ 4] seeks Fire - goes to exit opening 4

$17.9 \mathrm{~s}$ : $[12$ e $3 \circ 4 \times 1000$ y3500 p 3] reached exit opening 4

17.9s: $[12$ e 2 o 4 x 1000 y $3500 \mathrm{p}$ 3] found Fire

22.9 s: [12 e $2 \circ 4 \times 1000$ y3500 p 3] goes to Location e: 3 x:0 y:4500

22.9s: [12 e 2 o $4 \times 1000$ y3500 p 3] going to exit opening 4

22.9s: [12 e 2 o $4 \times 1000$ y3500 p 3] reached exit opening 4

22.9s: [12 e 3 o $4 \times 1000$ y3500 p 3] goes to Location $x: 0$ y: 4500

24.6s: [12 e $3 \circ 4 \mathrm{x} 0$ y $4500 \mathrm{p}$ 3] reached location

26.5s: [12 e $403 \times 500$ y6000 p 3 ] reaching end of stair flight

26.5s: [ 12 e 4 o $3 \times 500$ y $6000 \mathrm{p}$ 3] at end of stair flight travel

26.5s: [11 e 6 o $3 \times 500$ y6000 p 0 ] going to exit opening 7

29.6s: [12 e $304 \mathrm{x}$ o y4500 p 3] seeks Fire - goes to exit opening 4

$31.3 \mathrm{~s}:[12$ e $3 \circ 4 \times 1000$ y3500 p 3$]$ reached exit opening 4

31.3s: [12 e 2 o 4 x 1000 y $3500 \mathrm{p}$

$36.3 \mathrm{~s}$ : $[12$ e 2 o 4 x 1000 y3500 p

36.3s: $[12$ e 2 o $4 \times 1000$ y $3500 \mathrm{p}$

36.3s: [12 e 3 o 4 x 1000 y $3500 \mathrm{p}$

38.4s: $[11$ e 6 o 3 x 0 y6500 p

$38.4 \mathrm{~s}:[11$ e 6 o $3 \mathrm{x}$ 0 y6500 p

$38.8 \mathrm{~s}:[12$ e 3 o 4 x 1000 y $500 \mathrm{p}$

38.8s: $[12$ e 1 o 4 x 1000 y $500 \mathrm{p}$

3] found Fire

3] seeks others - goes to exit opening 4

3] reached exit opening 4

3] seeks others - goes to exit opening 2

0] reached exit opening 7

0] opening exit 7

3] reached exit opening 2

38.8s: [12 e $104 \times 1000$ y $500 \mathrm{p}$ 3] orders occupant 1 to evacuate

40.4s: Exit 7 on Level 1 is opened

40.4s: Occupant 3 has exited the building

$43.8 \mathrm{~s}$ : $[12$ e 1 o $2 \times 2500$ y $1500 \mathrm{p}$ 3] going to exit opening 2

43.8s: [12 e 1 o $1 \times 2500$ y1500 p 3 ] going to exit opening 2

$43.8 \mathrm{~s}$ : [ [12 e 1 o 4 x $1000 \mathrm{y} 500 \mathrm{p}$ 3] going to exit opening 2

43.8s: 112 e $104 \times 1000$ y $500 \mathrm{p}$ 3] reached exit opening 2

43.8s: [12 e 3 : 4 x 1000 y $500 \mathrm{p}$ 3] going to exit opening 6

45.9s: [12 e 1 o 2 x1000 y $500 \mathrm{p}$ 3] reached exit opening 2

45.9s: [12 e $1 \circ 1 \times 1000$ y 500 p 3$]$ reached exit opening 2

45.9s: [12 e 3 o 2 x1000 y $500 \mathrm{p} 3$ ] going to exit opening 6

47.4s: [12 e $101 \times 1000$ y $500 \mathrm{p}$ 3] next in queue

47.4s: [12 e $301 \times 1000$ y $500 \mathrm{p}$ 3] going to exit opening 6

48.8s: [12 e $3 \circ 4 \times 500$ y6000 p 3] reached exit opening 6

50.9s: [12 e $3 \circ 2 \times 500$ y6000 p 3 ] reached exit opening 6

50.9s: $[12$ e $402 \times 500$ y6000 p 2 ] going down the stairs

52.5s: $[12$ e $3 \circ 1 \times 500$ y6000 p 3$]$ reached exit opening 6

$52.5 \mathrm{~s}$ : [12 e $401 \times 500$ y6000 p 1] going down the stairs

(only warden is left now and begins communication with Master warden)

57.5s: [12 e $304 \times 500$ y6000 p 1] Confirmed Level 2 stair evacuation completed

57.5s: [12 e 3 o 4 x 500 y6000 p 1] Warden exiting Level 2

57.5s: Level 2 is empty

57.5s: $[12$ e $404 \times 500$ y6000 p 0$]$ going down the stairs

62.5s: Evacuating Level 1

$62.8 \mathrm{~s}$ : 112 e $402 \times 500$ y6000 p 2] reaching end of stair flight

62.8s: $[12$ e $402 \times 500 y 6000 \mathrm{p} 0]$ at end of stair flight travel

62.8s: [11 e $602 \times 500$ Y6000 p 0 ] going to exit opening 7

64.4s: $[12$ e $401 \times 500$ y $6000 \mathrm{p}$ 1] reaching end of stair flight

64.4s: [12 e $401 \times 500$ y $6000 \mathrm{p} 0$ ] at end of stair flight trave]

64.4s: [ 11 e $601 \times 500 y 6000 \mathrm{p} 0$ ] going to exit opening 7

69.4s: [12 e $404 \times 500$ y6000 p 0 ] reaching end of stair flight

69.4s: [12 e $404 \times 500 y 6000 \mathrm{p} 0$ ] at end of stair flight travel

69.4s: [11 e $604 \times 500$ y6000 p 0 ] going to exit opening 7

74.8s: [11 e 6 o $2 \times$ y y6500 p 0 ] reached exit opening 7

$74.8 \mathrm{~s}$ : Occupant 2 has exited the building

76.3s: [ 11 e 6 o $1 \mathrm{x} \quad 0 \mathrm{y} 6500 \mathrm{p}$ 0] reached exit opening 7

76.3 s: Occupant 1 has exited the building 
81.3s: [11 e $6 \circ 4 \mathrm{x} \quad 0 \mathrm{y} 6500 \mathrm{p} 0$ ] reached exit opening 7

$81.3 \mathrm{~s}$ : Occupant 4 has exited the building

81.3s: Simulation Completed - 11:01:40.69 (0.9 secs runtime)

$81.3 \mathrm{~s}: 4$ out of 4 evacuated successfully

A typical output line is described as follows:

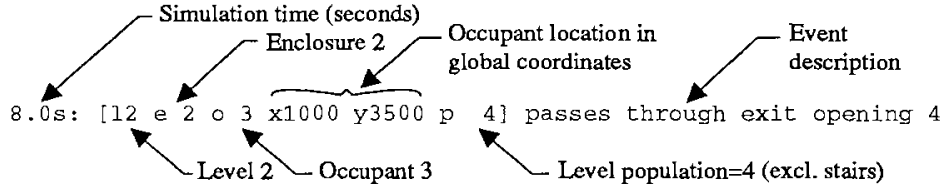

Notice that Occupant 1 (Management) did not act in accordance with the action defined in the Response Profile Table by going to investigate the fire upon becoming aware of it. The instruction from a Warden supersedes the intended action of the occupant in all cases. This example took 0.9 second to run on a $80486 \mathrm{DX}-33 \mathrm{mHz}$ personal computer. In comparison, in stage 1 of the validation process, a nine-story building with 2400 occupants took about 6 minutes.

\section{CURRENT LIMITATIONS AND FUTURE DEVELOPMENTS}

EvacSim is still being developed and due to the varied features of the program, its complete validation will be a lengthy process and is therefore being done in stages.

A number of human behavioural features which are more pertinent to occupants of residential or institutional buildings such as sleeping individuals, babies or elderly occupants who require assistance, are not presently modelled. In addition, although EvacSim enables the modelling of complex behavioural sequences, the data to structure such sequences is presently largely lacking.

In order to fully realise the potential of EvacSim in simulating the dynamic interaction of the occupants with the effects of fire in a building, it must be integrated with a fire model. This is the next major development stage. Following that, the effects of toxic products of combustion can then be modelled to assess the risk to life of exposed occupants.

\section{ACKNOWLEDGEMENTS}

This work was jointly funded by BHP Research and the Centre for Environmental Safety and Risk Engineering (CESARE) at Victoria University of Technology. The author would like to thank Professor Vaughan Beck of CESARE and Dr. Ian Thomas of BHP Research for their support and comments in this work.

\section{REFERENCES}

1. Bernard M. Levin, "EXITT - A Simulation Model of Occupant Decisions and Actions in Residential Fires", Fire Safety Science - Proceedings of the Second International Symposium, Tokyo, pp. 561-570, 1989. 
2. F.I. Stahl, "BFIRES-II A Behaviour Based Computer Simulation of Emergency Egress During Fires", Fire Technology, Vol. 18, No. 1, 1982.

3. G.N. Berlin, "A simulation model for assessing building fire safety", Fire Technology, Vol. 18, No. 1, pp. 66-76, 1982.

4. Filiz Ozel, "Simulation modelling of human behaviour in buildings", Simulation, June 1992.

5. R. Fahy, "EXIT89 - An Evacuation Model for High-Rise Buildings", Conference Proceedings of the Sixth International Interflam Conference, 1993.

6. V.M. Predtechenskii and A.I. Milinskii, Planning for Foot Traffic in Buildings, Amerind Publishing Company, Inc., New Delhi, India, 1978.

7. J.L. Pauls, "Effective-Width Model for Evacuation Flow in Buildings", Proceedings: Engineering Applications Workshop, Society of Fire Protection Engineers, Boston, 1980.

8. Harold E. Nelson and Hamish A. MacLennan, "Emergency Movement", SEPE Handbook of Fire Protection Engineering (P.J. DiNenno, ed.), National Fire Protection Association, Quincy, Massachusetts, 1988.

9. Fruin, J.J., Pedestrian Planning Design, Metropolitan Association of Urban Designers and Environmental Planners, Inc., New York, 1971.

10. Kiyoshi Takahashi, Takeyoshi Tanaka and Satoshi Kose, "An Evacuation Model for Use in Fire Safety Design of Buildings", Fire Safety Science - Proceedings of the Second International Symposium, Tokyo, pp. 551-560, 1989. 